\title{
Plasma and hepatic carnitine and coenzyme A pools in a patient with fatal, valproate induced hepatotoxicity
}

\author{
S Krähenbühl, G Mang, H Kupferschmidt, P J Meier, M Krause
}

\begin{abstract}
Reduced hepatic mitochondrial $\beta$-oxidation and changes in the plasma carnitine pool are important biochemical findings in valproate induced liver toxicity. The carnitine pools in plasma and liver and the liver coenzyme $A$ (CoA) pool in a patient with fatal, valproate induced hepatotoxicity were measured. In plasma and liver the free and total carnitine contents were decreased, whereas the ratios short chain acylcarnitine/total acid soluble carnitine were increased. The long chain acylcarnitine content was unchanged in plasma, and increased in liver. The total CoA content in liver was decreased by $84 \%$. This was due to reduced concentrations of CoASH, acetyl-CoA, and long chain acyl-CoA whereas the concentrations of succinylCoA and propionyl-CoA were both increased. The good agreement between the plasma and liver carnitine pools reflects the close relation between these two pools. The observed decrease in the hepatic CoASH and total CoA content has so far not been reported in humans with valproate induced hepatotoxicity and may be functionally significant.

(Gut 1995; 37: 140-143)
\end{abstract}

Keywords: carnitine, coenzyme A, liver toxicity, valproate.

Valproate is a branched chain fatty acid composed of eight carbons and has no structural relation to other antiepileptic drugs. Its antiepileptic properties were discovered by chance by Meunier et $a l,{ }^{1}$ and the drug was introduced into the anticonvulsant market in 1968 in Europe and 10 years later in the United States. ${ }^{2}$ Shortly after introduction, cases of severe and occasionally fatal hepatotoxicity in patients treated with valproate have been reported. ${ }^{2-4}$ The frequency of fatal valproate induced liver damage decreases with age, averaging roughly $1: 10000$ in children and 1:40 000 in adults, and is higher in patients receiving an antiepileptic polytherapy compared with monotherapy. ${ }^{4}$ The precise mechanism of valproate induced hepatotoxicity is currently not known. In vitro studies suggest that it is related to hepatic depletion of CoASH or microsomal production of metabolites such as 4-enevalproate and 2,4-ene-valproate, or both ${ }^{5}$; both mechanisms ultimately lead to decreased mitochondrial $\beta$-oxidation. In agreement with this concept, microvesicular steatosis, the principal histological finding in valproate induced hepatotoxicity, ${ }^{2-4}$ is also detected in other types of liver disease with decreased mitochondrial $\beta$-oxidation such as Reye's syndrome, Jamaican vomiting sickness, mitochondrial cytopathies, and acute fatty liver of pregnancy. ${ }^{5-7}$

It is well established that the plasma carnitine pool, which is believed to reflect changes in the hepatic carnitine and coenzyme A (CoA) pools, ${ }^{89}$ shows a shift towards short chain acylcarnitines when hepatic $\beta$-oxidation is decreased. ${ }^{10}$ This shift in the plasma carnitine pool has also been described in patients with severe valproate induced hepatotoxicity. ${ }^{11}$ In this paper, we characterise for the first time the liver carnitine and CoA pools in a patient with fatal valproate induced hepatotoxicity.

\section{Case report}

This 39 year old woman was known for connatal, bilateral ptosis, and progressive paralysis of external eye muscles. She had suffered from a manic depressive disorder since the age of 20 but was receiving no current medical treatment for this disease. Five months before the actual illness, she developed a frontal status epilepticus with convulsions, and was treated successfully with valproate $(2400 \mathrm{mg} /$ day $)$ and clonazepam (6 mg/day). A computer tomogram and magnetic resonance imaging of the brain showed multiple bifrontal and cerebellar lesions, suggesting a multi-infarct syndrome. Valproate and clonazepam serum concentrations were consistently in the therapeutic range, and the patient had initially a normal liver function (normal transaminase activity and no cholestasis). At the same time, treatment with aspirin (100 mg/day) was started. Four months after the start of the antiepileptic treatment, the patient developed progressive ataxia and became increasingly apathic. Treatment with carbamazepine (400 to $600 \mathrm{mg} /$ day) was started, which failed to improve the clinical status and had to be stopped because of leucopenia. The patient developed progressive anaemia and thrombocytopenia, the prothrombin index decreased, and the serum bilirubin concentration and transaminase activities increased. Coagulation studies were compatible with disseminated intravascular coagulation and the patient was admitted to hospital. At entry, the patient was somnolent and hypothermic $\left(34 \cdot 8^{\circ} \mathrm{C}\right)$, had a blood pressure of $85 / 60 \mathrm{~mm} \mathrm{Hg}$, a bilirubin 
concentration of $145 \mu \mathrm{M}$, the alanine transaminase activity was $35 \mathrm{U} / \mathrm{l}$, the aspartate transaminase activity $95 \mathrm{U} / \mathrm{l}$, the prothrombin ratio $39 \%$, the ammonia concentration $75 \mu \mathrm{M}$, and the thrombocyte count $47 \times 10^{9} / 1$. Hypofibrinogenaemia with increased fibrin degradation products, increased partial thromboplastine time and thrombocytopenia were compatible with disseminated intravascular coagulation. Epileptic signs were not detectable, and a computer tomogram of the brain was unchanged in comparison with the earlier investigations. The serum concentrations of clonazepam and valproate were in the therapeutic range and the antiepileptic treatment was initially continued. Blood and spinal fluid cultures were negative for bacterial growth. Twelve hours after entry, the patient developed a cardiovascular collapse and was resuscitated and intubated. Her subsequent course of illness was noticeable for deteriorating liver function with hepatorenal syndrome and progressive disseminated intravascular coagulation. The patient died five days after entry with multiorgan failure. Necropsy was performed five hours after death.

\section{Methods}

\section{Sample preparation}

Blood samples $(5 \mathrm{ml})$ were withdrawn in heparinised tubes, centrifuged at $600 \times g$ for five minutes, and the plasma was removed and frozen at $-20^{\circ} \mathrm{C}$ until analysis. The blood sample from the patient was obtained three days before death. The blood samples from control subjects were obtained after an overnight fast. Liver samples from the patient and from control subjects (different from control subjects used for the determination of plasma carnitine) were removed at necropsy (about five hours after death), and frozen at $-70^{\circ} \mathrm{C}$ until analysis. Control subjects died after an accident and had macroscopically and microscopically a normal liver.

\section{Assay methods}

Free, total acid soluble and long chain acylcarnitine concentrations in plasma and liver were determined by the radioenzymatic assay originally described by Cederblad ${ }^{12}$ with the modifications described by Brass and Hoppel. ${ }^{813}$ The method has coefficients of variation below $10 \% .^{13}$ Plasma and liver

TABLE I Plasma carnitine concentrations. Blood was withdrawn three days before death. The plasma carnitine concentration was determined by radioenzymatic analysis as described in the methods section. Carnitine concentrations are given as $\mu \mathrm{mol} /$. Data are presented as mean ( $95 \%$ confidence intervals).

\begin{tabular}{lcc}
\hline & Patient & $\begin{array}{c}\text { Controls } \\
(n=10)\end{array}$ \\
\hline Free carnitine & $18 \cdot 8$ & $35 \cdot 8(4 \cdot 0)$ \\
Short chain acylcarnitine (SCA Cn) & $5 \cdot 8$ & $5 \cdot 2(2 \cdot 8)$ \\
Total acid soluble carnitine (TAS Cn) & $24 \cdot 6$ & $41 \cdot 0(3 \cdot 2)$ \\
SCA Cn/TAS Cn & $0 \cdot 24$ & $0 \cdot 13(0 \cdot 05)$ \\
Long chain acylcarnitine & $2 \cdot 8$ & $3 \cdot 7(1 \cdot 1)$ \\
Total carnitine & $27 \cdot 4$ & $44 \cdot 7(7 \cdot 1)$ \\
\hline
\end{tabular}

TABLE II Characterisation of the liver carnitine pool.

Liver tissue was obtained at necropsy five hours after death. The carnitine content was determined by radioenzymatic analysis as described in the methods section. Carnitine concentrations are given as nmol/mg non-collagen protein. Data are presented as mean ( $95 \%$ confidence intervals).

\begin{tabular}{lll}
\hline & Patient & $\begin{array}{l}\text { Controls } \\
(n=3)\end{array}$ \\
\hline Free carnitine & $2 \cdot 98$ & $5 \cdot 51(0 \cdot 70)$ \\
Short chain acylcarnitine (SCA Cn) & $1 \cdot 19$ & $0 \cdot 82(0 \cdot 65)$ \\
Total acid soluble carnitine (TAS Cn) & $4 \cdot 17$ & $6 \cdot 33(0 \cdot 70)$ \\
SCA Cn/TAS Cn & $0 \cdot 29$ & $0 \cdot 13(0 \cdot 08)$ \\
Long chain acylcarnitine & $0 \cdot 80$ & $0 \cdot 48(0 \cdot 19)$ \\
Total carnitine & $4 \cdot 97$ & $6 \cdot 81(0 \cdot 48)$ \\
\hline
\end{tabular}

samples were prepared in perchloric acid and centrifuged for two minutes at $10000 \times \mathrm{g}$. In the supernatant, the free and the total acid soluble carnitine concentrations were determined. The short chain acylcarnitine concentration was calculated as the difference between the total acid soluble and the free carnitine concentrations. In the pellet, the long chain acylcarnitine content was determined. The total carnitine concentration was calculated as the sum of the total acid soluble and the long chain acylcarnitine concentrations.

The hepatic concentrations of $\mathrm{CoASH}$, short chain acyl-CoAs, and total acid soluble CoA were determined in neutralised perchloric acid extracts by high performance liquid chromatography (HPLC) as described previously. The total acid soluble CoA concentrations were determined after alkaline hydrolysis of the perchloric acid supernatants and the short chain acyl-CoA concentrations were calculated as the difference between the total acid soluble CoA and the CoASH concentrations. The long chain acyl-CoA concentrations were determined in the hydrolised pellet of the perchloric acid extracts by HPLC. ${ }^{14}$ The total CoA concentration was calculated by addition of the total acid soluble and the long chain acyl-CoA concentrations. The results obtained by HPLC (CoASH, total acid soluble CoA, and long chain acyl-CoA) were validated by the radioenzymatic method described by Cederblad. ${ }^{15}$ The results between the two methods differed by less than $10 \%$.

Liver non-collagen protein concentrations were determined according to Lowry ${ }^{16}$ with bovine serum albumin as a standard after alkaline hydrolysis of collagen. ${ }^{17}$ All analyses were performed in duplicate with the average of the two values reported in the result section. Results are given as mean (95\% confidence intervals).

\section{Results}

Histological examination of liver samples obtained at necropsy showed microvesicular steatosis, a finding that is compatible with valproate induced hepatopathy. Together with the clinical findings such as progressive ataxia and apathia, and disseminated intravascular coagulation, valproate toxicity was considered to be the most probable explanation for the rapid deterioration in this patient.

In plasma obtained three days before death, the free and total acid soluble carnitine, long 
TABLE III Characterisation of the liver coenzyme $A$ pool. Liver tissue was obtained at necropsy five hours after death. The coenzyme $A$ content was determined by high performance liquid chromatography and radioenzymatic analysis as described in the methods section. Coenzyme $A$ concentrations are given as pmol/mg non-collagen protein. Data are presented as mean (95\% confidence intervals).

\begin{tabular}{lcc}
\hline & Patient & $\begin{array}{l}\text { Controls } \\
(n=3)\end{array}$ \\
\hline CoASH & 101 & $1530(180)$ \\
Succinyl-CoA & 89 & $36(19)$ \\
Acetyl-CoA & 20 & $89(80)$ \\
Propionyl-CoA & 32 & $4(4)$ \\
Total acid soluble CoA & 278 & $1850(80)$ \\
Long chain acyl-CoA & 42 & $211(90)$ \\
Total CoA & 320 & $2060(130)$ \\
\hline
\end{tabular}

chain acylcarnitine, and total carnitine concentrations were decreased, and the short chain acylcarnitine concentration was unchanged (Table I). The ratio short chain acylcarnitine/total acid soluble carnitine was increased.

The hepatic non-collagen protein concentration was $50.3 \mathrm{mg} / \mathrm{g}$ in the patient and 113 (16) $\mathrm{mg} / \mathrm{g}$ in control subjects.

Table II shows the hepatic carnitine concentrations. In comparison with control livers, free, total acid soluble and total carnitine concentrations were decreased, the short chain acylcarnitine concentration was unchanged, and the long chain acylcarnitine concentration was increased. The short chain acylcarnitine/total acid soluble carnitine ratio was increased. Table III shows the hepatic CoA concentrations. With the exception of propionyl-CoA and succinyl-CoA, all other CoA species were decreased in the liver of the patient. In comparison with control livers, the hepatic CoASH content was decreased by $93 \%$ and the total CoA content by $84 \%$.

\section{Discussion}

It is well established that treatment with valproic acid can be associated with decreased free and total plasma carnitine concentrations in patients with $^{11}{ }^{18-20}$ or without hepatopathy. ${ }^{21-24}$ Valproate undergoes hepatic metabolism with the formation of acyl-CoAs, which can be converted to the corresponding acylcarnitines. ${ }^{525}$ In contrast with acyl-CoAs, acylcarnitines can be exported from the liver and can be excreted by the kidneys, causing significant carnitine losses, eventually leading to secondary carnitine deficiency. ${ }^{102627}$ In addition, it is known that the loss of free carnitine is also increased in patients excreting large amounts of acylcarnitines, as acylcarnitines such as valproylcarnitine can inhibit the renal reabsorption of filtered carnitine. ${ }^{202728}$ In comparison with the findings in our patient, the plasma carnitine concentrations in patients with alcohol induced liver disease ${ }^{29} 30$ or end stage primary biliary cirrhosis ${ }^{31}$ has been shown to be increased, suggesting that the plasma carnitine concentration in patients with liver disease depends on the cause of liver disease.

With the exception of the long chain acylcarnitines, which were increased in liver and not affected in plasma of our patient, the hepatic carnitine pool showed qualitatively similar changes as the plasma carnitine pool, supporting the concept that the liver and plasma carnitine pools interact closely. ${ }^{8}{ }^{9}$ As discussed, the decrease in the free and total carnitine content in the liver of our patient is therefore most probably a consequence of increased renal carnitine excretion. In agreement with our findings, short term administration of valproate to mice has been shown to decrease the free and total carnitine content and to increase the long chain acylcarnitine content in the liver, ${ }^{32-34}$ whereas longterm administration of valproate did not affect the hepatic carnitine pool in rats. ${ }^{35-37}$ In comparison, in patients or rats with liver cirrhosis, the total hepatic carnitine content seems to be affected by the cause of liver disease, showing a decrease in patients with end stage alcoholic liver cirrhosis, ${ }^{38}$ an increase in rats with secondary biliary cirrhosis, ${ }^{39}$ and no change in rats with carbon tetrachloride induced liver cirrhosis. ${ }^{40}$

The analysis of the hepatic CoA pool showed a considerable decrease in the CoASH and total CoA contents and increases in succinyl and propionyl-CoA. In comparison with our findings, short term administration of valproate to mice or rats, ${ }^{32} 3441$ isolated hepatocytes $^{41}$ or isolated mitochondria ${ }^{42}$ decreased the CoASH but not the total CoA content, and induced a shift from CoASH to acyl-CoAs. While the increase in the hepatic succinyl- and propionyl-CoA contents may be explained by the metabolism of valproate or by inhibition of hepatic mitochondrial metabolism, or both, ${ }^{52}$ the decrease in the total CoA content of our patient remains unexplained. Interestingly, the total CoA content in livers from rats with secondary biliary cirrhosis, an animal model of chronic liver disease with impaired hepatic fatty acid metabolism, ${ }^{43}$ was also found to be decreased. ${ }^{39}$ On the other hand, the hepatic coenzyme A pool in rats with carbon tetrachloride induced liver cirrhosis was not different from control rats (S Krähenbühl and E Brass, unpublished data), showing that the cause of liver disease is important for the induction of changes in the CoA pool. As CoASH is a critical substrate for $\beta$-oxidation, a decrease in the hepatic CoASH content could lead to impaired hepatic fatty acid metabolism, which is considered to represent the major mechanism in valproate induced hepatotoxicity. ${ }^{525}$

In contrast with the reversible inhibition of hepatic energy metabolism (including fatty acid oxidation), which is normally associated with the administration of valproate to humans or experimental animals, 532 35-3741 4244 fatal hepatic toxicity is rare. ${ }^{2-4}$ Two factors known to increase the risk of severe hepatic toxicity are concomitant administration of substances inducing hepatic cytochrome $\mathrm{P} 450$ isoenzymes and young age. ${ }^{4}$ Our findings support the concept that reduced hepatic mitochondrial metabolism represents another risk factor for valproate induced hepatotoxicity. ${ }^{28}$ Our patient was treated with low dose acetylsalicylic acid, a drug known to inhibit hepatic $\beta$-oxidation. ${ }^{45}$ In addition, our patient showed 
signs compatible with a disorder of mitochondrial energy metabolism such as ptosis and progressive external ophthalmoplegia. We therefore propose that valproic acid should not be given to epileptic patients with inborn deficiencies of fatty acid metabolism or to patients treated with drugs known to inhibit hepatic fatty acid oxidation, or both.

The authors thank Mrs B O'Neill for performing the carnitine and radioenzymatic coenzyme $A$ analyses, and $\mathrm{Dr} R$ Ha for performing the coenzyme A analysis by HPLC. The work was financially supported by grant 32-37280.93 from the Swiss National Science Foundation to S Krähenbühl.

1 Menuier H, Garroz G, Meunier Y, Eymard P, Aimard M. Proprietés pharmacodynamiques de l'acide n-dipropy18: $435-8$.

2 Zafrani ES, Berthelot P. Sodium valproate in the induction of unusual hepatotoxicity. Hepatology 1982; 2: 648-9.

3 Zimmermann HJ, Ishak KG. Valproate-induced hepatic injury: analyses of 23 fatal cases. Hepatology 1982; 2: 591-7.

4 Dreifuss FE, Santilli N, Langer DH, Sweeney KP, Moline KA, Menander KB. Valproic acid hepatic fatalities: a retrospective review. Neurology 1987; 37: 379-85.

5 Ponchaut S, Veitch K. Valproate and mitochondria. Biochem Pharmacol 1993; 46: 199-204.

6 Sherlock S, Dooley J. Diseases of the liver and biliary system. 9th ed. Oxford: Blackwell Scientific, 1992: $410-1$.

7 Bioulac-Sage P, Parrot-Roulaud F, Mazat JP, Lamireau T, Coquet M, Sandler B, et al. Fatal neonatal liver failure and mitochondrial cytopathy (oxidative phosphorylation deficiency): a light and electron microscopic study of the liver. Hepatology 1993; 18: 839-46.

8 Brass EP, Hoppel CL. Carnitine metabolism in the fasting rat. F Biol Chem 1978; 253: 2688-93.

9 Sandor A, Cseko J, Kispal G, Alkonyi I. Surplus acylcarnitines in the plasma of starved rats derive from the liver. f Biol Chem 1990; 265: 22313-6.

10 Stanley CA. New genetic defects in mitochondrial fatty acid oxidation and carnitine deficiency. Adv Pediatr 1987; 34: $59-88$

11 Bohan TP, Rogers P, Roe CR. Valproate and carnitine. In: Carter AL, ed. Current concepts in carnitine research. Boca Raton, Ann Arbor: CRC Press, 1992: 19-26.

12 Cederblad $G$, Lindstedt $S$. A method for the determination of carnitine in the picomole range. Clin Chim Acta 1972; 37: $235-43$.

13 Hoppel CL. Determination of carnitine. In: Techniques in diagnostic human biochemical genetics: a laboratory manual. New York: Wiley-Liss, 1991: 309-26.

14 Krähenbühl S, Brass EP. Inhibition of propionyl-CoA synthetase activity by organic acids. Reversal of prophanate inhibition of pyruvat $1991 ; 41$ : 1015-23.

15 Cederblad G, Carlin JI, Constantin-Teodosiu D, Harper P, Hultman PE. Radioisotopic assays of CoASH and carnitine and their acetylated forms in human skeletal muscle. Anal Biochem 1990; 185: 274-8.

16 Lowry OH, Rosenbrough NJ, Farr AL, Randall RJ. Protein measurement with the folin phenol reagent. $\mathcal{F}$ Biol Chem 1951; 193: 265-75.

17 Lilienthal JL, Zierler KL, Folk BP, Buka R, Riley MJ. A reference base and system for analysis of muscle constituents. $₹$ Biol Chem 1950; 182: 501-8.

18 Böhles $\mathrm{H}$, Richter $\mathrm{K}$, Wagner-Thiessen $\mathrm{E}$, Schäfer $\mathrm{H}$. Decreased serum carnitine in valproate induced Reye Decreased serum carnitine in valproate
syndrome. Eur $\mathcal{F}$ Pediatr 1982; 139: 185-6.

19 Matsuda I, Ohtani Y, Ninomiya N. Renal handling of carnitine in children with carnitine deficiency and hyperammonemia associated with valproate therapy. $\mathcal{f}$ Pediatr 1986; 109: 131-4.

20 Murakami K, Sugimoto T, Nishida N, Kobayashi Y, Kuhara T, Matsumoto I. Abnormal metabolism of carnitine and valproate in a case of acute encephalopathy during chronic valproate therapy. Brain Dev 1992; 14: 178-81.

21 Murphy JV, Marquardt KM, Shug AL. Vaproic acid associated abnormalities of carnitine metabolism. Lancet 1985; i: $820-1$

22 Laub MC, Paetzke-Brunner I, Jaeger G. Serum carnitine during valproic acid therapy. Epilepsia 1986; 27:

23 Triggs WJ, Bohan TP, Lin SN, Willmore J. Valproateinduced coma with ketosis and carnitine deficiency. Arch Neurol 1990; 47: 1131-3.

24 Hug G, McGraw CA, Bates SR, Landrigan EA. Reduction of serum carnitine concentrations during anticonvulsant therapy with phenobarbital, valproic acid, phenytoin, and carbamazepine in children. F Pediatr 1991; 119: 799-802.

25 Zarazepine in children. Pediatr 1991; 119: 799-802. kinetics of valproic acid - 1988. Clin Pharmacokinet 1988; 15: $367-89$.

26 Chalmers RA, Roe CR, Stacey TE, Hoppel CL. Urinary excretion of L-carnitine and acylcarnitines by patients with disorders of organic acid metabolism: evidence for secondary insufficiency of L-carnitine. Pediatr Res 1984; 18: $1325-8$.

27 Stanley CA, Berry CT, Bennett MJ, Willi SM, Treem WR, Hale DE. Renal handling of carnitine in secondary carnitine deficiency disorders. Pediatr Res 1993; 34: 89-97.

28 Tein I, DiMauro S, Xie ZW, De Vivo DC. Valproic acid impairs carnitine uptake in cultured human skin fibroblasts. An in vitro model for the pathogenesis of valproic acid-associated carnitine deficiency. Pediatr Res 1993; 34: $281-7$.

29 Fuller RK, Hoppel CL. Elevated plasma carnitine in hepatic cirrhosis. Hepatology 1983; 4: 554-8.

30 Fuller RK, Hoppel CL Plasma carnitine in alcoholism. Alcohol Clin Exp Res 1988; 12: 639-42.

31 Palambo JD, Borum PR, Jenkins RL, Trey C, Bistrian BR. Blood carnitine status after orthotopic liver transplantation in patients with end-stage liver disease. Am $\mathcal{F}$ Clin Nutr 1989; 50: 504-7.

32 Thurston JH, Carroll JE, Hauhart RE, Schiro JA. A single therapeutic dose of valproate affects liver carbohydrate, fat, adenylate, amino acid, coenzyme $\mathrm{A}$ and carnitine metabolism in infant mice: possible clinical significance. Life Sci 1985; 36: 1643-51.

33 Rozas I, Camina MF, Paz JM, Castro-Gago M, RodriguezSegade S. Effects of acute valproate administration on carnitine metabolism in mouse serum and tissues. Biochem Pharmacol 1990; 39: 181-5.

34 Thurston JH, Hauhart RE. Amelioration of adverse effects of valproic acid on ketogenesis and liver coenzyme A metabolism by cotreatment with pantothenate and carnitine in developing mice: possible clinical significance. Pediatr Res 1992; 31: 419-23.

35 Sugimoto T, Araki A, Nishida N, Sakane Y, Woo M, Takeuchi $\mathrm{T}$, et al. Hepatotoxicity in rat following administration of valproic acid: effect of L-carnitine supplementation. Epilepsia 1987; 28: 373-7.

36 Sugimoto T, Woo M, Nishida N, Takeuchi T, Sakame Y, Kabayashi Y. Hepatotoxicity in rat following administration of valproic acid. Epilepsia 1987; 28: 142-6.

37 Murakami K, Sugimoto T, Nishida N, Woo M, Araki A, Kobayashi Y, et al. Carnitine metabolism and morphometric change of liver mitochondria in valproate-treated rats. Neuropediatrics 1990; 21: 187-90.

38 Rudman D, Sewell CW, Ansley JD. Deficiency of carnitine in cachectic cirrhotic patients. F Clin Invest 1977; 60: in cachectic

39 Krähenbühl S, Brass EP. Fuel homeostasis and carnitine metabolism in rats with secondary biliary cirrhosis. Hepatology 1991; 14: 927-34.

40 Krähenbühl S, Weber FL, Brass EP. Decreased hepatic glycogen content and accelerated response to starvation in rats with carbon tetrachloride-induced cirrhosis. Hepatology 1991; 14: 1189-95.

41 Becker CM, Harris RA. Influence of valproic acid on hepatic carbohydrate and lipid metabolism. Arch Biochem Biophys 1983; 223: 381-92.

42 Ponchaut $S$, Van Hoof $F$, Veitch $K$. In vitro effects of valproate and valproate metabolites on mitochondrial oxidations. Relevance of $\mathrm{CoA}$ sequestration to the observed inhibitions. Biochem Pharmacol 1992; 43: 2435-42.

43 Krähenbühl S, Talos C, Reichen J. Mechanisms of impaired hepatic fatty acid metabolism in rats with long-term bile duct ligation. Hepatology 1994; 19: 1272-81.

44 Turnbull DM, Dick DJ, Wilson L, Sherratt HSA, Alberti KCMM. Valproate causes metabolic disturbance in normal man. I Neurol Neurosurg Psychiatry 1986; 49: 405-10.

45 Deschamps D, Fisch C, Fromenty B, Berson A, Degott C, Pessayre D. Inhibition by Salicylic acid of the activation and thus oxidation of long chain fatty acids. Possible role in the development of Reye's syndrome. $\mathcal{F}$ Pharmacol Exp Ther 1991; 259: 894-904. 